Pacific

Journal of

Mathematics

\title{
PROPER HOLOMORPHIC MAPS BETWEEN BOUNDED SYMMETRIC DOMAINS REVISITED
}

GAUTAM BHARALI AND JAIKRISHNAN JANARDHANAN 


\title{
PROPER HOLOMORPHIC MAPS BETWEEN BOUNDED SYMMETRIC DOMAINS REVISITED
}

\author{
GAUTAM BHARALI AND JAIKRISHNAN JANARDHANAN
}

\begin{abstract}
We prove that a proper holomorphic map between two nonplanar bounded symmetric domains of the same dimension, one of them being irreducible, is a biholomorphism. Our methods allow us to give a single, all-encompassing argument that unifies the various special cases in which this result is known. We discuss an application of these methods to domains having noncompact automorphism groups that are not assumed to act transitively.
\end{abstract}

\section{Introduction and statement of results}

The primary objective of this paper is to prove the following result:

Theorem 1.1. Let $D_{1}$ and $D_{2}$ be two bounded symmetric domains of complex dimension $n \geq 2$. Assume that either $D_{1}$ or $D_{2}$ is irreducible. Then, any proper holomorphic mapping of $D_{1}$ into $D_{2}$ is a biholomorphism.

This theorem is known in several special cases. For $D_{1}=D_{2}=\mathbb{B}^{n}$, the (Euclidean) ball in $\mathbb{C}^{n}$ with $n \geq 2$, the result was established by Alexander [1977]. This is a pioneering work that has motivated several generalizations to proper holomorphic maps between certain types of smoothly bounded pseudoconvex domains. Henkin and Novikov [1984] described a method for proving the result when $D_{1}=D_{2}=: D$ where $D$ is a bounded symmetric domain that is not of tube type. Later, Tsai [1993] established the result for $D_{1}$ and $D_{2}$ as above, provided $D_{1}$ is irreducible and $\operatorname{rank} D_{1} \geq \operatorname{rank} D_{2} \geq 2$.

Tsai's result is a broad metric-rigidity theorem (under the Bergman metric) for proper holomorphic maps of $D_{1}$ into $D_{2}$, where $D_{1}$ and $D_{2}$ are as above but not necessarily equidimensional. In such a result, the condition $\operatorname{rank} D_{1} \geq \operatorname{rank} D_{2} \geq 2$ is indispensable. Adapting Tsai's ideas to the equidimensional case, Tu [2002] established Theorem 1.1 in the higher-rank case, assuming $D_{1}$ is irreducible. In using Tsai's ideas, the assumption that $D_{1}$ is irreducible is essential — see [Tu 2002,

Bharali is supported by a UGC Centre for Advanced Study grant. Janardhanan is supported by a UGC Centre for Advanced Study grant and by a scholarship from the IISc.

MSC2010: primary 32H02, 32M15; secondary 32H40.

Keywords: bounded symmetric domains, Harish-Chandra realization, Jordan triple systems, proper holomorphic maps, rigidity, Schwarz lemma. 
Proposition 3.3] — and it is not clear that a small mutation of those ideas allows one to weaken this assumption. In our work, we are able to assume either $D_{1}$ or $D_{2}$ to be irreducible precisely by not relying too heavily on the fine structure of these domains. Indeed, we wish to emphasize that the focus of this work is not on mopping up the residual cases in Theorem 1.1. The methods in [Henkin and Novikov 1984] (which rely on [Tumanov and Khenkin 1982]), [Tsai 1993] and [Tu 2002] are tied, in a rather maximalistic way, to the fine structure of a bounded symmetric domain. In contrast, we present some ideas that make very mild use of the underlying orbit structure of the bounded symmetric domains. They could therefore be applied to manifolds whose automorphism groups are not assumed to act transitively but are merely "large enough". Theorem 1.5 is an illustration of this notion. These ideas also provide a unified argument, irrespective of rank or reducibility, for Theorem 1.1.

We need to be more precise about the preceding remarks. This requires some elaboration on the objects of interest. A bounded symmetric domain in $\mathbb{C}^{n}$ is the holomorphic imbedding in $\mathbb{C}^{n}$ of some Hermitian symmetric space of noncompact type. It is irreducible if it is not a product of bounded symmetric domains of lower dimension. Cartan studied Hermitian symmetric spaces of noncompact type and classified the irreducible ones, showing that they are one of six types of homogeneous spaces. An outcome of [Harish-Chandra 1956] is that these homogeneous spaces (and products thereof) can be imbedded in $\mathbb{C}^{n}$ as bounded convex balanced domains (we say that a domain $D \subset \mathbb{C}^{n}$ is balanced if, for any $z \in D$, we have $\zeta z \in D$ for each $\zeta$ in the closed unit disc centered at $0 \in \mathbb{C}$ ). This imbedding is unique up to a linear isomorphism of $\mathbb{C}^{n}$. Such a realization of a bounded symmetric domain is called a Harish-Chandra realization.

The three main features that we wish to emphasize about this work are:

(a) The arguments in [Alexander 1977] involve estimates showing how a proper mapping maps conical regions with vertex on $\partial \mathbb{B}^{n}$ into the "admissible" approach regions of Korányi [1969]. Boundary approach, in a somewhat different sense, plus Chern-Moser theory [1974] make an appearance in [Tumanov and Khenkin 1982]. In contrast, apart from, and owing to, a result of Bell [1982] on boundary behavior, our proof involves rather "soft" methods.

(b) The techniques underlying [Tsai 1993] and [Tu 2002] rely almost entirely on the fine structure of a bounded symmetric domain. Specifically, they involve studying the effect of a proper holomorphic map on the characteristic symmetric subspaces of a bounded symmetric domain of rank at least 2 . In contrast, our techniques rely on only a coarse distinction between the different strata that comprise the boundary of an irreducible bounded symmetric domain (e.g., see Remark 4.8 below). 
(c) An advantage of arguments that rely on only a coarse resolution of a bounded symmetric domain is that some of them are potentially applicable to the study of domains that have noncompact automorphism groups but are not assumed to be symmetric. A demonstration this viewpoint is the proof of Theorem 1.5 below.

Let $D$ be a bounded symmetric domain. The main technical tool that facilitates our study of the structure of $\partial D$, and describes certain elements of $\operatorname{Aut}(D)$ with the optimal degree of explicitness, is the notion of Jordan triple systems. The application of Jordan triple systems to geometry appears to have been pioneered by Koecher [1999]. Our reference on this subject is the lecture notes of Loos [1977], which are devoted specifically to bounded symmetric domains. Jordan triple systems and versions of the Schwarz lemma are our primary tools. We present next an outline of how we use these tools.

An important lemma, which is inspired by Alexander's work, is the following.

Key Lemma 1.2. Let $D$ be a realization of an irreducible bounded symmetric domain of dimension $n \geq 2$ as a bounded convex balanced domain in $\mathbb{C}^{n}$. For $z \in D \backslash\{0\}$, let $\Delta_{z}:=\{\zeta z: \zeta \in \mathbb{C}$ and $\zeta z \in D\}$. Let $W_{1}$ and $W_{2}$ be two regions in $D$ such that $0 \in W_{1} \cap W_{2}$ and let $F: D \rightarrow D$ be a holomorphic map. Assume that:

(i) $F$ maps $W_{1}$ biholomorphically onto $W_{2}$ with $F(0)=0$.

(ii) There exists a nonempty open set $U \subset W_{1} \backslash\{0\}$ such that, for each $z \in U$, $\Delta_{z} \subset W_{1}$ and $\Delta_{F(z)} \subset W_{2}$.

Then, $F$ is an automorphism of $D$.

This is a consequence of Vigué's Schwarz lemma [1991] (see Result 4.6 below), and the irreducibility of $D$ is essential to the lemma.

Our proof of Theorem 1.1 may be summarized as follows (we will assume here that $D_{1}$ and $D_{2}$ are Harish-Chandra realizations of the domains in question):

- By Bell's theorem [1982, Theorem 2], $F$ extends to a neighborhood of $\bar{D}_{1}$ and we can find a point $p$ in the Bergman-Shilov boundary of $D_{1}$, and a small ball $B$ around it, such that $\left.F\right|_{B}$ is a biholomorphism.

- We may assume that $F(0)=0$. Let $\left\{a_{k}\right\}$ be a sequence in $D_{1} \cap B$ converging to $p$ and let $b_{k}:=F\left(a_{k}\right)$. Let $\phi_{k}^{j} \in \operatorname{Aut}\left(D_{j}\right)$ be an automorphism that maps 0 to $a_{k}$ if $j=1$ and to $b_{k}$ if $j=2$. It turns out that both $p$ and $F(p)$ are peak points, whence $\phi_{k}^{j} \rightarrow p^{(j)}$ uniformly on compact subsets, where $p^{(1)}:=p$ and $p^{(2)}:=F(p)$.

- Using the Schwarz lemma for convex balanced domains (Result 4.5 below) we show that a subsequence of $\left\{\left(\phi_{j}^{2}\right)^{-1} \circ F \circ \phi_{j}^{1}\right\}$ converges to a linear map and that, owing to the tautness of $D_{1}$ and $D_{2}$, this map is a biholomorphism of $D_{1}$ onto $D_{2}$. 
- We may now take $D_{1}=D_{2}=D$. We shall use our Key Lemma 1.2 with $W_{1}=\left(\phi_{k}^{1}\right)^{-1}(D \cap B)$ and $W_{2}=\left(\phi_{k}^{2}\right)^{-1}(D \cap F(B))$ for $k$ sufficiently large.

- Since the analytic discs $\Delta_{z}$ and $\Delta_{F(z)}$ are not relatively compact in $D$, the mode of convergence of $\left\{\phi_{k}^{j}\right\}$ isn't a priori good enough to infer that appropriate families of these discs will be swallowed up by $W_{j}, j=1,2$. By Bell's theorem, each $\phi_{k}^{j}$ extends to some neighborhood of $D$. We show that $\left\{\phi_{k}^{j}\right\}$, passing to a subsequence and relabeling if necessary, converges uniformly on certain special circular subsets of $D$ that are adherent to $\partial D$. This is enough to overcome the difficulty just described.

Let us define a term that we used in the sketch above, which we shall also need in stating our next theorem.

Definition 1.3. Let $D \varsubsetneqq \mathbb{C}^{n}$ be a domain and let $p \in \partial D$. We say that $p$ is a peak point if there exists a function $h \in \mathbb{O}(D) \cap \mathscr{C}(\bar{D} ; \mathbb{C})$ such that $h(p)=1$ and $|h(z)|<1$ for all $z \in \bar{D} \backslash\{p\}$. The function $h$ is called a peak function for $p$.

When a domain $D$ is bounded, the noncompactness of $\operatorname{Aut}(D)$ (in the compactopen topology) is equivalent to $D$ having a boundary orbit-accumulation point; see [Narasimhan 1971].

Definition 1.4. Let $D \varsubsetneqq \mathbb{C}^{n}$ be a domain and let $p \in \partial D$. We say that $p$ is a boundary orbit-accumulation point if there exist a point $a \in D$ and a sequence of automorphisms $\left\{\phi_{k}\right\}$ of $D$ such that $\lim _{k \rightarrow \infty} \phi_{k}(a)=p$.

With the last two definitions, we are in a position to state our second theorem. Note that $D_{1}$ is not assumed to be a bounded symmetric domain. Yet, some of the techniques sketched above (versions of which have been used to remarkable effect in the literature in this field) are general enough to be applicable to the following situation.

Theorem 1.5. Let $D_{1}$ be a bounded convex balanced domain in $\mathbb{C}^{n}$ whose automorphism group is noncompact and let $p$ be a boundary orbit-accumulation point. Let $D_{2}$ be a realization of a bounded symmetric domain as a bounded convex balanced domain in $\mathbb{C}^{n}$. Assume that there is a neighborhood $U$ of $p$ and a biholomorphic map $F: U \rightarrow \mathbb{C}^{n}$ such that $F\left(U \cap D_{1}\right) \subset D_{2}$ and $F\left(U \cap \partial D_{1}\right) \subset \partial D_{2}$. Assume that either $p$ or $F(p)$ is a peak point. Then, there exists a linear map that maps $D_{1}$ biholomorphically onto $\mathrm{D}_{2}$.

Remark 1.6. Theorem 1.5 (together with Bell's theorem [1982]) gives a very short proof of the rigidity theorem of Mok and Tsai [1992] under the additional assumption that the convex domain $D$ in their result is also circular. There is extensive literature on rigidity theorems relating to bounded symmetric domains, but we shall not dwell any further on it. 
Remark 1.7. A version of this result can be proved without assuming that $D_{1}$ is either balanced or convex. $D_{1}$ merely needs to be complete Kobayashi hyperbolic. However, in this case, the biholomorphism of $D_{1}$ onto $D_{2}$ will not, in general, be linear. We prefer the above version: the conclusion that there exists a linear equivalence places Theorem 1.5 among the rigidity theorems alluded to in Remark 1.6.

The layout of this paper is as follows. Since Jordan triple systems play a vital role in describing not just the structure of the boundary of a bounded symmetric domain, but also some of its key automorphisms, we begin with a primer on Jordan triple systems. Readers who are familiar with Jordan triple systems can skip to Section 3, where we discuss the boundary geometry of bounded symmetric domains. Section 4 is devoted to stating and proving certain propositions that are essential to our proofs. Finally, in Sections 5 and 6, we present the proofs of the results stated above.

\section{A primer on Jordan triple systems}

There is a natural connection between bounded symmetric domains and certain Hermitian Jordan triple systems. This section collects several definitions and results that are required to give a coherent description of the boundary of a bounded symmetric domain (which we shall discuss in the next section).

Unless otherwise stated, the results in this section can be found in the UC Irvine lectures by Loos [1977] describing how Jordan triple systems can be used to study the geometry of bounded symmetric domains.

Definition 2.1. A Hermitian Jordan triple system is a complex vector space $V$ endowed with a triple product $(x, y, z) \mapsto\{x, y, z\}$ that is symmetric and bilinear in $x$ and $z$ and conjugate-linear in $y$ and satisfies the Jordan identity

$$
\begin{aligned}
\{x, y,\{u, v, w\}\}-\{u, v,\{x, y, w\}\}=\{\{x, y, u\}, v, w\}- & \{u,\{y, x, v\}, w\} \\
& \text { for all } x, y, u, v, w \in V .
\end{aligned}
$$

Such a system is said to be positive if for each $x \in V \backslash\{0\}$ for which $\{x, x, x\}=\lambda x$ (where $\lambda$ is a scalar), we have $\lambda>0$.

As mentioned in Section 1, a bounded symmetric domain of complex dimension $n$ has a realization $D$ as a bounded convex balanced domain in $\mathbb{C}^{n}$. Let $\left(z_{1}, \ldots, z_{n}\right)$ be the global holomorphic coordinates coming from the product structure on $\mathbb{C}^{n}$ and let $\left(\boldsymbol{\epsilon}_{1}, \ldots, \boldsymbol{\epsilon}_{n}\right)$ denote the standard ordered basis of $\mathbb{C}^{n}$. Let $K_{D}$ denote the Bergman kernel of (the above realization of) $D$ and $h_{D}$ the Bergman metric. The function $\{\cdot, \cdot, \cdot\}: \mathbb{C}^{n} \times \mathbb{C}^{n} \times \mathbb{C}^{n} \rightarrow \mathbb{C}^{n}$ obtained by the requirement

$$
h_{D}\left(\left\{\boldsymbol{\epsilon}_{i}, \boldsymbol{\epsilon}_{j}, \boldsymbol{\epsilon}_{k}\right\}, \boldsymbol{\epsilon}_{l}\right)=\left.\frac{\partial^{4} \log K_{D}(z, z)}{\partial z_{i} \partial \bar{z}_{j} \partial z_{k} \partial \bar{z}_{l}}\right|_{z=0},
$$


and by extending $\mathbb{C}$-linearly in the first and third variables and $\mathbb{C}$-antilinearly in the second, has the property that $\left(\mathbb{C}^{n},\{\cdot, \cdot, \cdot\}\right)$ is a positive Hermitian Jordan triple system (abbreviated hereafter as PHJTS). This relationship is a one-to-one correspondence between finite-dimensional PHJTSs and bounded symmetric domains which we shall make more precise in Section 3.

Let $(V,\{\cdot, \cdot, \cdot\})$ be an HJTS. It will be convenient to work with the operators

$$
\mathbf{D}(x, y) z=\mathbf{Q}(x, z) y:=\{x, y, z\} .
$$

We define the operator $Q: V \rightarrow \operatorname{End}(V)$ by $Q(x) y:=\mathbf{Q}(x, x) y / 2$. For any $x \in V$, we can define the so-called odd powers of $x$ recursively by

$$
x^{(1)}:=x \quad \text { and } \quad x^{(2 p+1)}:=Q(x) x^{(2 p-1)} \quad \text { if } p \geq 1 .
$$

A vector $e \in V$ is called a tripotent if $e^{(3)}=e$.

Tripotents are important to this discussion because:

- A finite-dimensional PHJTS has plenty of nonzero tripotents.

- Given a finite-dimensional PHJTS $(V,\{\cdot, \cdot, \cdot\})$, any vector $V$ has a certain canonical decomposition as a linear combination of tripotents.

- In a finite-dimensional PHJTS, the set of tripotents forms a real-analytic submanifold.

We refer the interested reader to [Loos 1977, Chapter 3] for details of the first fact. As for the second fact, we need a couple of new notions. First, given an HJTS $(V,\{\cdot, \cdot, \cdot\})$, we say that two tripotents $e_{1}, e_{2} \in V$ are orthogonal if $\mathrm{D}\left(e_{1}, e_{2}\right)=0$. Second, given $x \in V$, we define the real vector space $\ll x \gg$ by

$$
\ll x \gg:=\operatorname{span}_{\mathbb{R}}\left\{x^{(2 p+1)}: p=0,1,2, \ldots\right\} .
$$

These two notions allow us to state the following:

Result 2.2 (spectral decomposition theorem). Let $(V,\{\cdot, \cdot, \cdot\})$ be a finite-dimensional PHJTS. Then, each $x \in V \backslash\{0\}$ can be written uniquely as

$$
x=\lambda_{1} e_{1}+\cdots+\lambda_{s} e_{s},
$$

where $\lambda_{1}>\lambda_{2}>\cdots>\lambda_{s}>0$ and $\left\{e_{1}, \ldots, e_{s}\right\}$ is an $\mathbb{R}$-basis of $\ll x \gg$ comprising pairwise orthogonal tripotents.

The decomposition of $x \in V$ as given by Result 2.2 is called the spectral decomposition of $x$. The assignment $x \mapsto \lambda_{1}(x)$, where $\lambda_{1}(x)$ is as given by (2-3), is a well-defined function and can be shown to be a norm on $V$. This norm is called the spectral norm on $V$.

Next, we present another decomposition, which gives us the second ingredient needed to describe the boundary geometry of a bounded symmetric domain. 
Result 2.3 (Pierce decomposition). Let $(V,\{\cdot, \cdot, \cdot\})$ be an HJTS and let $e \in V$ be a tripotent. Then, the spectrum of $\mathrm{D}(e, e)$ is a subset of $\{0,1,2\}$. Let

$$
V_{j}=V_{j}(e):=\{x \in V: \mathrm{D}(e, e) x=j x\}, \quad j \in \mathbb{Z} .
$$

Then:

(a) $V=V_{0} \oplus V_{1} \oplus V_{2}$.

(b) If $e \neq 0$, then $e \in V_{2}$.

(c) We have the relation $\left\{V_{\alpha}, V_{\beta}, V_{\gamma}\right\} \subset V_{\alpha-\beta+\gamma}$.

(d) $V_{0}, V_{1}$ and $V_{2}$ are Hermitian Jordan subsystems of $\{\cdot, \cdot, \cdot\}$.

The direct-sum decomposition (a) given by this result is called the Pierce decomposition of $V$ with respect to the tripotent $e$. The ideas that go into proving the Pierce decomposition theorem allow us to construct a special partial order on the set of tripotents of $V$. In order to avoid statements that are vacuously true, unless stated otherwise, we take $(V,\{\cdot, \cdot, \cdot\})$ to be a PHJTS. Let $e, e^{\prime} \in V$ be tripotents. We say that $e$ is dominated by $e^{\prime}\left(e \preceq e^{\prime}\right)$ if there is a tripotent $e_{1}$ orthogonal to $e$ such that $e^{\prime}=e+e_{1}$. We say that $e$ is strongly dominated by $e^{\prime}\left(e \prec e^{\prime}\right)$ if $e \preceq e^{\prime}$ and $e \neq e^{\prime}$. The result of interest, in this regard, is the following:

Result 2.4. Let $(V,\{\cdot, \cdot, \cdot\})$ be an HJTS. Let $e_{1}, e_{2} \in V$ be orthogonal tripotents and let $e=e_{1}+e_{2}$. If $e^{\prime} \in V$ is a tripotent orthogonal to $e$, then $e^{\prime}$ is orthogonal to $e_{1}$ and $e_{2}$.

Now suppose $\{\cdot, \cdot, \cdot\}$ is positive. Then, the relation $\preceq$ is a partial order on the set of tripotents.

Definition 2.5. A tripotent is said to be minimal (or primitive) if it is minimal for $\preceq$ among nonzero tripotents. It is said to be maximal if it is maximal for $\preceq$.

Result 2.6. Consider the tripotents of $V$ partially ordered by $\preceq$.

(1) A tripotent e is maximal if and only if the Pierce space $V_{0}(e)$ equals 0.

(2) If, for a tripotent e, the Pierce space $V_{2}(e)$ equals $\mathbb{C} e$, then e is primitive.

Let us now also assume that $(V,\{\cdot, \cdot, \cdot\})$ is finite-dimensional. Given any nonzero tripotent $e$, it follows from finite-dimensionality and the repeated application of Result 2.4 that $e$ can be written as a sum of mutually orthogonal primitive tripotents. This brings us to the final concept in this primer: the rank of a tripotent $e$ is the minimum number of primitive tripotents required for such a decomposition of $e$, while the rank of $(V,\{\cdot, \cdot, \cdot\})$ is the highest rank that a tripotent of $V$ can have. 


\section{The boundary geometry of bounded symmetric domains}

In this section we describe the boundary of a bounded symmetric domain in terms of the positive Hermitian Jordan triple system associated to it. Thus, we shall follow the notation introduced in Section 2. Recall that a bounded symmetric domain $D$ has a realization as a bounded convex balanced domain. When we say "Hermitian Jordan triple system associated to $D$ ", it is implicit that $D$ is this realization and the association is the one given by (2-1). This is a one-to-one correspondence, described as follows:

Result 3.1 [Loos 1977, Theorem 4.1]. Let D be a realization of a bounded symmetric domain as a bounded convex balanced domain in $\mathbb{C}^{n}$ for some $n \in \mathbb{Z}_{+}$. Then, $D$ is the open unit ball in $\mathbb{C}^{n}$ with respect to the spectral norm determined by the PHJTS associated to D. Conversely, given a PHJTS $\left(\mathbb{C}^{n},\{\cdot, \cdot, \cdot\}\right)$, the open unit ball with respect to the spectral norm determined by it is a bounded symmetric domain D, and the PHJTS associated to D by the rule $(2-1)$ is $\left(\mathbb{C}^{n},\{\cdot, \cdot, \cdot\}\right)$.

In what follows, whenever we mention a bounded symmetric domain $D$, it will be understood that $D$ is a bounded convex balanced realization.

The boundary of a bounded symmetric domain $D \subset \mathbb{C}^{n}$ has a certain stratification into real-analytic submanifolds that can be described in terms of the PHJTS associated to $D$. The first part of this section is devoted to describing this stratification. Fix a bounded symmetric domain $D \subset \mathbb{C}^{n}$ and let $\left(\mathbb{C}^{n},\{\cdot, \cdot, \cdot\}_{D}\right)$ be the PHJTS associated to it. It turns out (see [Loos 1977, Theorem 5.6]) that the set $M_{D}$ of tripotents of $\mathbb{C}^{n}$ with respect to $\{\cdot, \cdot, \cdot\}_{D}$ is a disjoint union of real-analytic submanifolds of $\mathbb{C}^{n}$. For each $e \in M_{D}$, let $M_{D, e}$ denote the connected component of $M_{D}$ containing $e$. The tangent space $T_{e}\left(M_{D, e}\right)$, viewed extrinsically (i.e., so that $e+T_{e}\left(M_{D, e}\right)$ is the affine subspace of all tangents to $M_{D, e}$ at $\left.e\right)$, is

$$
T_{e}\left(M_{D, e}\right)=i A(e) \oplus V_{1}(e),
$$

where $A(e)$ is determined by the relation $V_{2}(e)=\left\{x+i y \in \mathbb{C}^{n}: x, y \in A(e)\right\}$ and $V_{j}(e)$ is the eigenspace of $j=0,1,2$ in the Pierce decomposition of $\mathbb{C}^{n}$ with respect to $e$.

Let $M_{D}^{*}$ be the set of all nonzero tripotents and let $\|\cdot\|_{D}$ denote the spectral norm determined by $\{\cdot, \cdot, \cdot\}_{D}$. Define

$$
\begin{aligned}
E_{D} & :=\left\{(e, v) \in \mathbb{C}^{n} \times \mathbb{C}^{n}: e \in M_{D}^{*} \text { and } v \in V_{0}(e)\right\}, \\
\mathfrak{B}_{D} & :=\left\{(e, v) \in E_{D}:\|v\|_{D}<1\right\} .
\end{aligned}
$$

We can write $\mathfrak{B}_{D}$ as a disjoint union of the form

$$
\mathfrak{B}_{D}:=\bigsqcup_{\alpha \in \mathscr{C}} \mathfrak{B}_{D, \alpha},
$$


where $\mathscr{C}$ is the set of connected components of $M_{D}^{*}$, and each $\mathfrak{B}_{D, \alpha}$ is a connected, real-analytic submanifold of $\mathbb{C}^{n} \times \mathbb{C}^{n}$ that is a real-analytic fiber bundle whose fibers are unit $\|\cdot\|_{D}$-discs. The key theorem about the boundary of $D$ is as follows:

Result 3.2 [Loos 1977, Chapter 6]. Let $D$ be a bounded symmetric domain in $\mathbb{C}^{n}$ and let $\mathbf{f}: \mathfrak{B}_{D} \rightarrow \mathbb{C}^{n}$ be defined by $\mathbf{f}(e, v):=e+v$. Then:

(i) $\mathbf{f}_{\mathfrak{B}_{D, \alpha}}$ is an imbedding for each $\alpha \in \mathscr{C}$.

(ii) $\partial D=\bigsqcup_{\alpha \in \mathscr{\ell}} M_{D, \alpha}$, where $\mu_{D, \alpha}:=\mathbf{f}\left(\mathfrak{B}_{D, \alpha}\right)$.

(iii) In the above stratification of $\partial D$, if $M_{D, \alpha}$ is of dimension $d_{\alpha}$, then it is a closed, connected, real-analytic imbedded submanifold of the open set

$$
\mathbb{C}^{n} \backslash \bigcup_{\beta: \operatorname{dim}_{\mathbb{R}}\left(\mu_{D, \beta}\right)<d_{\alpha}} \mu_{D, \beta} .
$$

Furthermore, when $D$ is an irreducible bounded symmetric domain in $\mathbb{C}^{n}$, then we can provide further information. Here, the rank of a bounded symmetric domain is the rank of the Jordan triple system $\left(\mathbb{C}^{n},\{\cdot, \cdot, \cdot\}_{D}\right)$.

Result 3.3 [Loos 1977, Chapter 6; Vigué 1991, Théorème 7.3]. Let D be an irreducible bounded symmetric domain in $\mathbb{C}^{n}$ of rank $r$, and let $\mathscr{b}$ denote the set of connected components of $\mathfrak{B}_{D}$. Then:

(i) $\mathscr{C}$ has cardinality $r$.

(ii) Each connected component of the decomposition (3-1) is a bundle over a submanifold of nonzero tripotents of rank $j, j \in\{1, \ldots, r\}$. Denoting this bundle as $\mathfrak{B}_{D, j}, j \in\{1,2, \ldots, r\}$, we can express the stratification of $\partial D$ given by Result 3.2 (ii) as

$$
\partial D=\bigsqcup_{j=1}^{r} M_{D, j},
$$

where $\mathcal{M}_{D, j}:=\mathbf{f}\left(\mathfrak{B}_{D, j}\right)$, and each $\mathcal{M}_{D, j}$ is connected.

(iii) The stratum $\mu_{D, 1}$ is dense in $\partial D$.

The other goal of this section is to describe the structure of the germs of complexanalytic varieties contained in the boundary of a bounded symmetric domain $D$. This structure can be described in extremely minute detail; see, for instance, [Wolf 1972]. In fact, the papers about higher-rank bounded symmetric domains mentioned in Section 1 make extensive use of this fine structure. However, in this work, we only need very coarse information about the complex analytic structure of $\partial D$, specifically, the distinction between the Bergman-Shilov boundary of $D$ and its complement in $\partial D$.

We denote the Bergman-Shilov boundary of $D$ by $\partial_{S} D$. We shall not formally define here the notion of the Shilov boundary of a uniform algebra; we shall merely 
state that the Bergman-Shilov boundary of a bounded domain $D \Subset \mathbb{C}^{n}$ is the Shilov boundary of the uniform algebra $A(D):=\mathscr{O}(D) \cap \mathscr{C}(\bar{D})$. However, we do carefully state the following definition:

Definition 3.4. Let $D$ be a bounded domain in $\mathbb{C}^{n}$. An affine $\partial D$-component is an equivalence class under the equivalence relation $\sim_{A}$ on $\partial D$ given by

$x \sim_{A} y \Longleftrightarrow x$ and $y$ can be joined by a chain of segments lying in $\partial D$,

where a segment is a subset of $\mathbb{C}^{n}$ of the form $\{u+t v: t \in(0,1)\}, u, v \in \mathbb{C}^{n}$. A holomorphic arc component of $\partial D$ is an equivalence class under the equivalence relation $\sim_{H}$ on $\partial D$ given by

$x \sim_{H} y \Longleftrightarrow x$ and $y$ can be joined by a chain of analytic discs lying in $\partial D$.

Roughly speaking, given a bounded domain $D \Subset \mathbb{C}^{n}$ and a point $x \in \partial D$, the holomorphic arc component of $\partial D$ containing $x$ is the largest (germ of a) complexanalytic variety lying in $\partial D$ that contains $x$. The information that we require about holomorphic boundary components is:

Result 3.5 [Loos 1977, Theorem 6.3]. Let D be the realization of a bounded symmetric domain as a bounded convex balanced domain in $\mathbb{C}^{n}$.

(i) The affine $\partial D$-components and the holomorphic arc components of $\partial D$ coincide.

(ii) A boundary component containing a point $x \in \partial D$ is a nonempty open region in some $\mathbb{C}$-affine subspace of positive dimension passing through $x$ unless $x$ is a maximal tripotent.

Finally, we mention the following description of the Bergman-Shilov boundary of a bounded symmetric domain:

Result 3.6 [Loos 1977, Theorem 6.5]. Let $D \Subset \mathbb{C}^{n}$ be as in Result 3.5. The Bergman-Shilov boundary of D coincides with each of the following sets:

(i) the set of maximal tripotents of $\mathbb{C}^{n}$ with respect to $\{\cdot, \cdot, \cdot\}_{D}$;

(ii) the set of extreme points of $\bar{D}$;

(iii) the set of points of $\bar{D}$ having the maximum Euclidean distance from $0 \in \mathbb{C}^{n}$.

\section{Some essential propositions}

This section contains several lemmas and propositions - some being simple consequences of known results, and some requiring substantial work - that will be needed to prove our theorems. We begin with the following result of Bell: 
Result 4.1 [Bell 1982, Theorem 2]. Suppose $f: D_{1} \rightarrow D_{2}$ is a proper holomorphic map between bounded circular domains. Suppose further that $D_{2}$ contains the origin and that the Bergman kernel $K(w, z)$ associated to $D_{1}$ is such that for each compact subset $G$ of $D_{1}$, there is an open set $U=U(G)$ containing $\bar{D}_{1}$ such that $K(\cdot, z)$ extends to be holomorphic on $U$ for each $z \in G$. Then $f$ extends holomorphically to a neighborhood of $\bar{D}_{1}$.

Now let $D$ be any bounded balanced domain (not necessarily convex) in $\mathbb{C}^{n}$. If $D$ is not convex, it will not be a unit ball with respect to some norm on $\mathbb{C}^{n}$. However, we do have a function that has the same homogeneity property as a norm, with respect to which $D$ is the "unit ball". The function $M_{D}: \mathbb{C}^{n} \rightarrow[0, \infty)$ defined by

$$
M_{D}(z):=\inf \{t>0: z / t \in D\}
$$

is called the Minkowski functional for D. Assume that the intersection of each complex line passing through $0 \in \mathbb{C}^{n}$ with $\partial D$ is a circle. Let $G$ be a compact subset of $D$. Then, as $M_{D}$ is upper semicontinuous, there exists $r_{G} \in(0,1)$ such that $G \subset\left\{z \in \mathbb{C}^{n}: M_{D}(z)<r_{G}\right\}$ and the latter is an open set. Hence $z / r_{G} \in D$ for all $z \in G$. Clearly, $r_{G} w \in D$ for all $w \in\left\{z \in \mathbb{C}^{n}: M_{D}(z)<1 / r_{G}\right\}=: U(G)$. By our assumptions, $\bar{D} \subset U(G)$. Let $K_{D}$ be the Bergman kernel of $D$. We recall that

$$
K_{D}(w, z)=\sum_{v \in \mathbb{N}} \psi_{v}(w) \overline{\psi_{v}(z)} \text { for all }(w, z) \in D \times D,
$$

where the right-hand side converges absolutely and uniformly on any compact subset of $D \times D$ and $\left\{\psi_{v}\right\}_{v \in \mathbb{N}}$ is any complete orthonormal system for the Bergman space of $D$. Then - owing to the fact that the collection $\left\{C_{\alpha} z^{\alpha}: \alpha \in \mathbb{N}^{n}\right\}$ (where $C_{\alpha}>0$ are suitable normalization constants) is a complete orthonormal system for the Bergman space of $D$ - we can infer two things. First, the functions

$$
\phi_{z}(w):=K_{D}\left(r_{G} w, z / r_{G}\right), \quad w \in U(G),
$$

are well-defined by power series for each $z \in G$. Secondly,

$$
K_{D}\left(r_{G} w, z / r_{G}\right)=K_{D}(w, z) \text { for all }(w, z) \in D \times G .
$$

Comparing this with (4-1), we see that each $\phi_{z}$ extends $K_{D}(\cdot, z)$ holomorphically. In view of Result 4.1, we have just deduced:

Lemma 4.2. Let $f: D_{1} \rightarrow D_{2}$ be a proper holomorphic map between bounded circular domains. Suppose $D_{1}$ and $D_{2}$ are both balanced. Assume that the intersection of every complex line passing through 0 with $\partial D_{1}$ is a circle. Then $f$ extends holomorphically to a neighborhood of $\bar{D}_{1}$.

We remark that the above conclusion also follows from [Bell 1993].

Let $D$ be a bounded symmetric domain in $\mathbb{C}^{n}$. Let $\left(\mathbb{C}^{n},\{\cdot, \cdot, \cdot\}_{D}\right)$ be the Jordan triple system associated to $D$ (as in other places in this paper, we assume that $D$ 
is a Harish-Chandra realization). Let $\mathrm{D}_{D}$ and $Q_{D}$ be the maps (2-2) for the triple product $\{\cdot, \cdot, \cdot\}_{D}$. We define the linear operators $\mathrm{B}_{D}(x, y): \mathbb{C}^{n} \rightarrow \mathbb{C}^{n}$, where

$$
\mathbf{B}_{D}(x, y):=\mathrm{id}_{D}-\mathbf{D}_{D}(x, y)+Q(x) Q(y), \quad x, y \in \mathbb{C}^{n} .
$$

Consider the sesquilinear form $(x, y) \mapsto \operatorname{Tr}\left[\mathbf{D}_{D}(x, y)\right]$ on $\mathbb{C}^{n}$. It turns out that the positivity of $\{\cdot, \cdot, \cdot\}_{D}$ is equivalent to this sesquilinear form being an inner product on $\mathbb{C}^{n}$; see [Loos 1977, Chapter 3]. Furthermore with respect to this inner product, we have

$$
\mathrm{B}_{D}(x, y)^{*}=\mathrm{B}_{D}(y, x) \text { for all } x, y \in \mathbb{C}^{n} .
$$

It is now easy to deduce that $\mathrm{B}_{D}(a, a)$ is a self-adjoint, positive semidefinite linear operator. Consequently, $\mathrm{B}_{D}(a, a)$ admits a unique positive semidefinite square root, which we denote by $\mathrm{B}_{D}(a, a)^{1 / 2}$. Having made these two definitions, we can state the following useful facts about the geometry of $D$.

Result 4.3 [Loos 1977, Proposition 9.8; Roos 2000, Proposition III.4.1]. Let D be the realization of a bounded symmetric domain as a convex balanced domain in $\mathbb{C}^{n}$. Fix a point $a \in D$ and let

$$
g_{a}(z):=a+\mathrm{B}_{D}(a, a)^{1 / 2}\left(\mathrm{id}_{D}+\mathrm{D}_{D}(z, a)\right)^{-1}(z) \text { for all } z \in D .
$$

Then, $g_{a} \in \operatorname{Aut}(D), g_{a}(0)=a$, and $g_{a}^{\prime}(z)=\mathrm{B}_{D}(a, a)^{1 / 2} \circ \mathrm{B}_{D}(z,-a)^{-1}$. Furthermore, $g_{a}^{-1}=g_{-a}$.

Various versions of the following lemma have been known for a long time. We refer the reader to [Rudin 1980, Lemma 15.2.2] for a proof.

Lemma 4.4. Let $D$ be a bounded domain in $\mathbb{C}^{n}$ and let $p \in \partial D$. Assume that there exists a ball $B$ centered at $p$ and a function $h \in \mathbb{O}(B \cap D) \cap \mathscr{C}(\overline{B \cap D} ; \mathbb{C})$ such that $h(p)=1$ and $|h(z)|<1$ for all $z \in \overline{B \cap D} \backslash\{p\}$. Let $a_{0} \in D$ and $\left\{\phi_{k}\right\}$ be a sequence of automorphisms of $D$ such that $\phi_{k}\left(a_{0}\right) \rightarrow p$ as $k \rightarrow \infty$. Then, $\left\{\phi_{k}\right\}$ converges uniformly on compact subsets of $D$ to const $_{p}$ - the map that takes the constant value $p$.

We now state a version of Schwarz's lemma for convex balanced domains and then a version of Schwarz's lemma for irreducible bounded symmetric domains, both of which are needed in the proof of our Key Lemma 1.2 (see Section 1).

Result 4.5 [Rudin 1980, Theorem 8.1.2]. Let $\Omega_{1}$ and $\Omega_{2}$ be balanced regions in $\mathbb{C}^{n}$ and $\mathbb{C}^{m}$ respectively, and let $F: \Omega_{1} \rightarrow \Omega_{2}$ be a holomorphic map. Suppose $\Omega_{2}$ is convex and bounded. Then:

(i) $F^{\prime}(0)$ maps $\Omega_{1}$ into $\Omega_{2}$.

(ii) $F\left(r \Omega_{1}\right) \subseteq r \Omega_{2}(0<r \leq 1)$ if $F(0)=0$. 
Result 4.6 [Vigué 1991, Théorème 7.4]. Let D be an irreducible bounded symmetric domain in $\mathbb{C}^{n}$ in its Harish-Chandra realization (whence it is the unit ball in $\mathbb{C}^{n}$ for the associated spectral norm $\|\cdot\|)$. Let $F: D \rightarrow D$ be a holomorphic map such that $F(0)=0$. Assume that for some nonempty open set $U \subset D$, we have $\|F(z)\|=\|z\|$ for all $z \in U$. Then $F$ is an automorphism of $D$.

With these two results, we can now give a proof of our Key Lemma 1.2:

The proof of the Key Lemma 1.2. Let $z \in U$, and set $w:=F(z)$. By hypothesis, $F$ maps $\Delta_{z}$ into $D$ and $\left(\left.F\right|_{W_{1}}\right)^{-1}$ maps $\Delta_{w}$ into $D$. Applying Result 4.5 to $\left.F\right|_{\Delta_{z}}$ and to $\left.\left(\left.F\right|_{W_{1}}\right)^{-1}\right|_{\Delta_{w}}$, we have $\|F(z)\|=\|z\|$ for every $z \in U$. Thus by the Schwarz lemma for irreducible bounded symmetric domains, $F$ is an automorphism of $D$.

We now state and prove a technical proposition regarding the invertibility of the operator $\mathrm{B}_{D}$ at certain off-diagonal points in $\partial D \times \partial D$, where $D$ is an irreducible bounded symmetric domain of dimension at least 2 . Here $M_{D, 1}$ denotes the stratum of $\partial D$ described by Result 3.3. This result and our Key Lemma 1.2 are the central ingredients in the proof of our main theorem.

Proposition 4.7. Let $D$ be the realization of an irreducible bounded symmetric domain of dimension $n$ as a bounded convex balanced domain in $\mathbb{C}^{n}, n \geq 2$. Let $p \in \partial D$. For each $z_{0} \in M_{D, 1}$ and each $M_{D, 1}$-open neighborhood $U \ni z_{0}$, there exists a point $w \in U$ such that $\operatorname{det} \mathrm{B}_{D}(\cdot, p)$ is nonzero on the set $\{\zeta w: \zeta \in \mathbb{C},|\zeta|=1\}$.

Remark 4.8. In the following proof, we argue by assuming that the conclusion is false. We can instantly arrive at a contradiction at the point $(\bullet)$ in the proof below if we invoke results on the fine structure of $\partial D$; see [Wolf and Korányi 1965] or [Wolf 1972], for instance. However, we provide an elementary argument beyond (•) to complete the proof in the hope that appropriate analogues of Proposition 4.7 may be formulated in other contexts.

Proof. Let us denote det $\mathrm{B}_{D}(z, p)$ by $h(z)$, where $z \in \mathbb{C}^{n}$. Let us assume that the result is false. Then, there exist a point $z_{0} \in \mathcal{M}_{D, 1}$ and an $\mathcal{M}_{D, 1}$-open neighborhood $U \ni z_{0}$ such that for each $w \in U$, there exists a $\zeta_{w} \in\{\zeta \in \mathbb{C}:|\zeta|=1\}$ with $h\left(\zeta_{w} w\right)=0$. Let $q$ denote the quotient map $q: \mathbb{C}^{n} \backslash\{0\} \rightarrow \mathbb{C} \mathbb{P}^{n-1}$. Also write

$$
Z_{h}:=h^{-1}\{0\}, \quad Z:=Z_{h} \cap M_{D, 1} .
$$

Our assumption implies that $q(Z)$ contains a nonempty open set $\mathscr{V} \subset \mathbb{C} \mathbb{P}^{n-1}$. Let $\mathscr{A}:=\left\{z \in \mathbb{C}^{n}: 1-\varepsilon<\|z\|<1+\varepsilon\right\}$, where $\|\cdot\|$ denotes the spectral norm relative to which $D$ is the unit ball and $\varepsilon$ is a fixed number in $(0,1)$. As $\mathscr{V} \subset q(\mathscr{A})$, it is easy to see that $\mathscr{V}$ can be covered by finitely many holomorphic coordinate patches $\left(U_{1}, \psi_{1}\right), \ldots,\left(U_{M}, \psi_{M}\right)$ such that the maps

$$
q_{j}:=\left.\psi_{j} \circ q\right|_{q^{-1}\left(U_{j}\right) \cap \mathscr{A}}: q^{-1}\left(U_{j}\right) \cap \mathscr{A} \rightarrow \mathbb{C}^{n-1}
$$


are Lipschitz maps. Since Lipschitz maps cannot increase Hausdorff dimension (see [Rudin 1980, Proposition 14.4.4], for instance) and $\operatorname{dim}_{\mathbb{R}}(\mathscr{V})=2 n-2$, the preceding discussion shows that the Hausdorff dimension of $Z$ (and hence the dimension of $Z$ as a real-analytic set) is $2 n-2$. As $Z_{h}$ is a complex analytic subvariety, its singular locus is of complex dimension at most $n-2$. Thus, we can find a point $x_{0} \in Z$ that is a regular point of $Z_{h}$ and an open ball $B$ around $x_{0}$ that is so small that:

- $M_{D, 1} \cap B$ is a submanifold of $B$;

- $B \cap Z_{h}$ is an $(n-1)$-dimensional complex submanifold of $B$;

- the dimension of $B \cap Z$ is $2 n-2$.

These three facts imply that $M:=B \cap Z_{h} \subset M_{D, 1}$. We can deduce this by considering a local defining function $\rho_{B}: B \rightarrow \mathbb{R}$ for $\mathcal{M}_{D, 1}$ and observing that, by Łojasiewicz's theorem [1959], $\left.\rho_{B}\right|_{M} \equiv 0$. If $D=\mathbb{B}^{n}$, we already have a contradiction and, hence, the proof.

Since $\mu_{D, 1}$ is a real-analytic submanifold of $\mathbb{C}^{n} \backslash \bigsqcup_{j \geq 2} \mu_{D, j}$, where $\mu_{D, j}$ are the strata of $\partial D$ discussed in Section 3, we can define the Levi-form of $M_{D, 1}$ denoted by $\mathfrak{L}(z, V)$, where $z \in M_{D, 1}, V \in H_{z}\left(M_{D, 1}\right)$. A few words about notation: in this proof, we shall work with the tangent bundle of $M_{D, 1}$ defined extrinsically. So, when referring to vectors in $T_{z}\left(M_{D, 1}\right)$, we shall view them either as real or as complex vectors, as convenient, such that $z+T_{z}\left(\mu_{D, 1}\right)$ is the hyperplane tangent to $M_{D, 1}$ at $z \in M_{D, 1}$. In this scheme,

$$
H_{z}\left(M_{D, 1}\right):=T_{z}\left(M_{D, 1}\right) \cap i T_{z}\left(M_{D, 1}\right) .
$$

As $\operatorname{dim}_{\mathbb{C}}(M)=n-1$, we have $\mathfrak{L}(z, \cdot) \equiv 0$ for all $z \in M$. The curve $\gamma:(-\varepsilon, \varepsilon) \rightarrow$ $M_{D, 1}$ (for $\varepsilon>0$ suitably small), $\gamma(t):=\exp (i t) z$, is transverse to $M$ at $z$. This is because if $\gamma^{\prime}(0)=i z$ were in $H_{z}\left(M_{D, 1}\right)$, then

$$
i \gamma^{\prime}(0)=-z \in H_{z}\left(M_{D, 1}\right) \subset T_{z}\left(M_{D, 1}\right),
$$

which contradicts the convexity of $D$. Consequently, for $\varepsilon_{0}>0$ sufficiently small, the set $\left\{\exp (i t) z: t \in\left(-\varepsilon_{0}, \varepsilon_{0}\right), z \in M\right\}$ contains an $M_{D, 1}$-open neighborhood of $x_{0}$. Thus, $M_{D, 1}$ is Levi-flat at $x_{0}$. As $M_{D, 1}$ is real-analytic, it is a Levi-flat hypersurface.

We shall now show that the Levi-flatness of $M_{D, 1}$ leads to a contradiction. Let us pick an $x \in \mathcal{M}_{D, 1}$. Owing to Levi-flatness, we can find a ball $B_{x}$, centered at $x$, such that

$$
D_{x}^{-}:=D \cap B_{x}, \quad D_{x}^{+}:=B_{x} \backslash \bar{D}
$$

are both pseudoconvex. Let $\boldsymbol{n}_{x}$ denote the unit outward normal vector to $\partial D$ at $x$ $\left(x \in M_{D, 1}\right)$. Owing to convexity of $D$, we can find an $\varepsilon_{0}>0$ and a $\delta_{0}>0$ such that

$$
H_{x}\left(\varepsilon_{0} ; \delta\right):=x+\delta \boldsymbol{n}_{x}+\left\{V \in H_{x}\left(\mathcal{M}_{D, 1}\right):|V|<\varepsilon_{0}\right\} \subset D_{x}^{+}
$$


for each $\delta \in\left(0, \delta_{0}\right)$. Here, $|\cdot|$ denotes the Euclidean norm. As $H_{x}\left(\varepsilon_{0} ; \delta\right)$ is a copy of a complex $(n-1)$-dimensional ball and as $D_{x}^{+}$is taut - see [Kerzman and Rosay 1981, Proposition 2.1] - it follows that $H_{x}\left(\varepsilon_{0} ; 0\right) \subset \mathcal{M}_{D, 1}$. To summarize, $\mathcal{M}_{D, 1}$ has the following property:

(•) At each $x \in M_{D, 1}$, a germ of the set $\left(x+H_{x}\left(M_{D, 1}\right)\right)$ lies in $M_{D, 1}$.

Let us now pick and fix a point $y^{0} \in M_{D, 1}$. Let $\left(z_{1}, \ldots, z_{n}\right)$ be global holomorphic coordinates in $\mathbb{C}^{n}$, associated to an appropriate rigid motion of $D$, such that $y^{0}=(0, \ldots, 0), D \subset\left\{\operatorname{Re} z_{1}>0\right\}$ and $H_{y^{0}}\left(M_{D, 1}\right)=\left\{z_{1}=0\right\}$ relative to these coordinates. Let $W$ be a nonzero vector in $H_{y^{0}}\left(M_{D, 1}\right)$ and let $D_{W}:=D \cap \operatorname{span}_{\mathbb{C}}\left\{W, \boldsymbol{n}_{y^{0}}\right\}$. Clearly, $D_{W}$ is convex and by $(\bullet), \mathcal{M}_{D, 1} \cap \operatorname{span}_{\mathbb{C}}\left\{W, \boldsymbol{n}_{y^{0}}\right\}=: \mathcal{M}_{W}$ has the property that for each point $y \in \mathcal{M}_{W}$, the germ of a complex line through $y$, call it $\Lambda_{y, W}$, lies in $\mathcal{M}_{W}$. Let us view $D_{W}$ as lying in $\mathbb{C}^{2}$, whence a portion of $\mathcal{M}_{W}$ near $(0,0)$ can be parametrized by three real variables as follows:

$$
r(t, u, v)=\rho(t)+a(t)(u+i v), \quad|t|<\varepsilon_{1}, \quad|u|,|v|<\varepsilon_{2},
$$

where $\rho:\left(-\varepsilon_{1}, \varepsilon_{1}\right) \rightarrow M_{W}$ is a smooth curve through $(0,0)$ such that $\rho^{\prime}(t)$ is orthogonal to $\Lambda_{\rho(t), W}$ for each $t$, and $a:\left(-\varepsilon_{1}, \varepsilon_{1}\right) \rightarrow \mathbb{C}^{2}$ is such that $a(t)$ is parallel to $\Lambda_{\rho(t), W}$ for each $t$. For the remainder of this paragraph, $\boldsymbol{n}(t, u, v)$ will denote the inward unit normal to $\partial D_{W}$ at $r(t, u, v)$, and $\cdot$ will denote the standard inner product on $\mathbb{R}^{4}$. Define the matrix-valued function $\Gamma:\left(-\varepsilon_{1}, \varepsilon_{1}\right) \times\left(-\varepsilon_{2}, \varepsilon_{2}\right)^{2} \rightarrow \mathbb{R}^{3 \times 3}$ by

$$
\Gamma(\tau, U, V):=\left.\operatorname{Hess}_{t, u, v}(r(t, u, v) \cdot \boldsymbol{n}(\tau, U, V))\right|_{(t, u, v)=(\tau, U, V)} .
$$

The convexity of $D_{W}$ implies that $\Gamma(\tau, U, V)$ is positive semidefinite at each $(\tau, U, V)$ (recall that $\boldsymbol{n}(\tau, U, V)$ is the inward normal at $r(\tau, U, V)$ ). By choosing $\varepsilon_{1}, \varepsilon_{2}>0$ small enough, we can ensure that $\left(n_{1}^{2}+n_{2}^{2}\right)(t, u, v) \neq 0$ for every $(t, u, v)$, where we write $\boldsymbol{n}=\left(n_{1}, n_{2}, n_{3}, n_{4}\right)$, and that $a$ is of the form $a(t)=(\alpha(t)+i \beta(t), 1)$. We compute to observe that two of the principal minors of $\Gamma$ turn out to be $-\left(n_{1} \alpha^{\prime}+n_{2} \beta^{\prime}\right)^{2}$ and $-\left(n_{2} \alpha^{\prime}-n_{1} \beta^{\prime}\right)^{2}$, which must be nonnegative. This gives us the system of equations

$$
\begin{aligned}
n_{1} \alpha^{\prime}+\left.n_{2} \beta^{\prime}\right|_{(\tau, U, V)} & =0 \\
-n_{1} \beta^{\prime}+\left.n_{2} \alpha^{\prime}\right|_{(\tau, U, V)} & =0 \text { for all }(\tau, U, V) .
\end{aligned}
$$

By our assumption on $\boldsymbol{n}$, this implies that $\alpha^{\prime}=\beta^{\prime} \equiv 0$. Restating this geometrically, there is a small $M_{W}$-open neighborhood of $0 \in \partial D_{W}$ such that, for every $y$ in this neighborhood, $\Lambda_{y, W}$ is parallel to the vector $W$. This holds true for each nonzero $W \in H_{y^{0}}\left(M_{D, 1}\right)$. Thus, there is an $M_{D, 1}$-open patch $\omega \ni y^{0}$ such that

$$
x+H_{x}\left(M_{D, 1}\right) \text { is parallel to }\left\{z_{1}=0\right\} \text { for every } x \in \omega .
$$


By Result 3.3, $\mu_{D, 1}$ is connected. Thus, if $y^{0} \neq y \in \mathcal{M}_{D, 1}$, then $y$ can be joined to $y^{0}$ by a chain of $\mathcal{M}_{D, 1}$-open patches $\omega_{0}, \ldots, \omega_{N}$, where $\omega_{0}$ equals the patch $\omega$ in (4-2), $\omega_{j-1} j \cap \omega_{j} \neq \varnothing, j=1, \ldots, N$, and $\omega_{N} \ni y$. By a standard argument of real-analytic continuation, we deduce that (4-2) holds with $\omega_{N}$ replacing $\omega$ (where $z_{1}$ comes from the global system of coordinates fixed at the beginning of the previous paragraph). Hence, $x+H_{x}\left(M_{D, 1}\right)$ is parallel to $\left\{z_{1}=0\right\}$ for each $x \in M_{D, 1}$. As $M_{D, 1}$ is dense in $\partial D$ and $D$ is bounded, we can find a $\xi \in D$ and a vector $W=\left(W_{1}, \ldots, W_{n}\right)$ with $W_{1}=0$ such that the ray $\{\xi+t W: t \geq 0\}$ intersects $\partial D$ at a point in $M_{D, 1}$. Then, this ray must be tangential to $M_{D, 1}$ at the point of intersection, which is absurd as $D$ is convex. Hence, our initial assumption must be false.

\section{The proof of Theorem 1.1}

Before we proceed further, we clarify our notation for the different norms that will be used in the proof of Theorem 1.1. With $D_{1}$ and $D_{2}$ as in Theorem 1.1, $\|\cdot\|_{j}$ will denote the spectral norm such that $D_{j}$ is the unit $\|\cdot\|_{j}$-ball in $\mathbb{C}^{n}, j=1,2$. The Euclidean norm on $\mathbb{C}^{n}$ will be denoted by $|\cdot|$. We will also need to impose norms on certain linear operators on $\mathbb{C}^{n}$. We shall use the operator norm induced by the Euclidean norm: for a $\mathbb{C}$-linear operator $A$ on $\mathbb{C}^{n}$, we set

$$
\|A\|_{\text {op }}:=\sup _{|x|=1}|A x| .
$$

Proof of Theorem 1.1. We shall take $D_{1}$ and $D_{2}$ to be Harish-Chandra realizations of the given bounded symmetric domains. We may assume, composing $F$ with suitable automorphisms if necessary, that $F(0)=0$.

By Lemma 4.2, $F$ extends to a holomorphic map defined on a neighborhood $N$ of $\bar{D}_{1}$. For simplicity of notation, we shall denote this extension also by $F$. The complex Jacobian $\operatorname{Jac}_{\mathbb{C}} F$ is holomorphic on $N$ and $\operatorname{Jac}_{\mathbb{C}} F \not \equiv 0$ on $D_{1}$. Hence, by the maximum principle, $\operatorname{Jac}_{\mathbb{C}} F \not \equiv 0$ on $\partial D_{1}$. By definition, we can find a point $p \in \partial_{S} D_{1}$ such that

$$
\sup _{\bar{D}_{1}}\left|\operatorname{Jac}_{\mathbb{C}} F\right|=\left|\operatorname{Jac}_{\mathbb{C}} F(p)\right| \neq 0 .
$$

By the inverse function theorem, we can find a ball $B(p, r) \subset N$ such that $\left.F\right|_{B(p, r)}$ is injective. Let us write

$$
\Omega_{1}:=B(p, r) \cap D_{1}, \quad \Omega_{2}:=F(B(p, r)) \cap D_{2} .
$$

We shall use our Key Lemma 1.2 (see Section 1 and its proof in Section 4) to deduce the result. The regions $W_{1}$ and $W_{2}$ of that lemma will be constructed by applying suitable automorphisms to $\Omega_{1}$ and $\Omega_{2}$.

Claim.

$$
F(p) \in \partial_{S} D_{2} .
$$


Suppose $F(p) \notin \partial_{S} D_{2}$. It follows from Result 3.5 and Result 3.6 that there are a vector $V \in \mathbb{C}^{n} \backslash\{0\}$ and neighborhood $\omega$ of $0 \in \mathbb{C}$ such that $\psi(\omega) \subset F(B(p, r)) \cap \partial D_{2}$, where $\psi: \omega \ni \zeta \mapsto F(p)+\zeta V$. Next, define

$$
\widetilde{\psi}:=\left(\left.F\right|_{B(p, r)}\right)^{-1} \circ \psi \text {. }
$$

Since $\left.F\right|_{D_{1}}$ is proper and $\left.F\right|_{B(p, r)}$ is injective,

$$
F(z) \in F(B(p, r)) \cap \partial D_{2} \Longleftrightarrow z \in B(p, r) \cap \partial D_{1} .
$$

Thus $\widetilde{\psi}(\omega) \subset \partial D_{1}$. Furthermore, $\widetilde{\psi}$ is nonconstant and $\widetilde{\psi}(0)=p$. By definition, each point of $\widetilde{\psi}(\omega) \backslash\{p\}$ lies in the holomorphic arc component of $\partial D_{1}$ containing $p$. This is a contradiction since $p$, being an extreme point, is a one-point affine $\partial D_{1}$ component and thus, by Result 3.5, a one-point holomorphic arc component of $\partial D_{1}$. Hence the claim.

Let us now take a sequence $\left\{a_{k}\right\} \subset \Omega_{1}$ such that $a_{k} \rightarrow p$, and let $b_{k}:=F\left(a_{k}\right)$. Let $\phi_{k}^{1} \in \operatorname{Aut}\left(D_{1}\right)$ denote an automorphism that maps 0 to $a_{k}$. Let $\phi_{k}^{2} \in \operatorname{Aut}\left(D_{2}\right)$ be an automorphism that maps 0 to $b_{k}$. Owing to Result 3.6 and to convexity, we can construct a peak function for $p$ on $\bar{D}_{1}$. Likewise (in view of the last claim) $F(p)$ is a peak point of $D_{2}$. By Lemma 4.4, we get

$$
\phi_{k}^{j} \rightarrow \text { const }_{p^{j}} \text { uniformly on compacts, } \quad j=1,2,
$$

where $p^{1}:=p$ and $p^{2}:=F(p)$.

We now define

$$
\Omega_{j}^{k}:=\left(\phi_{k}^{j}\right)^{-1}\left(\Omega_{j}\right), \quad j=1,2, k \in \mathbb{Z}_{+} .
$$

Given any $r>0$, write $r D_{j}:=\left\{z \in \mathbb{C}^{n}:\|z\|_{j}<r\right\}, j=1,2$. By (5-1), there exists a sequence $k_{1}<k_{2}<k_{3}<\cdots$ in $\mathbb{Z}_{+}$such that

$$
\phi_{k_{v}}^{1}\left((1-1 / s) \bar{D}_{1}\right) \subset \Omega_{1} \quad \text { for all } v \geq s, s \in \mathbb{Z}_{+} .
$$

By (5-1) again, we can extract a sequence of indices $v(1)<v(2)<v(3)<\cdots$ such that

$$
\phi_{k_{v(t)}}^{2}\left((1-1 / s) \bar{D}_{2}\right) \subset \Omega_{2} \quad \text { for all } t \geq s, s \in \mathbb{Z}_{+} .
$$

In the interest of readability of notation, let us reindex $\left\{k_{v(s)}\right\}_{s \in \mathbb{Z}_{+}}$as $\left\{k_{m}\right\}_{m \in \mathbb{Z}_{+}}$. Then, the above can be summarized as

(*) With the sequences of maps $\left\{\phi_{k}^{1}\right\} \subset \operatorname{Aut}\left(D_{1}\right)$ and $\left\{\phi_{k}^{2}\right\} \subset \operatorname{Aut}\left(D_{2}\right)$ as described above, there are a sequence $\left\{k_{m}\right\}_{m \in \mathbb{Z}_{+}} \subset \mathbb{Z}_{+}$and a strictly increasing $\mathbb{Z}_{+}$-valued function $v^{*}$ such that

$$
\begin{aligned}
(1-1 / s) \bar{D}_{1} \subset \Omega_{1}^{k_{m}} & \text { for all } m \geq s, s \in \mathbb{Z}_{+}, \\
\left(1-1 / v^{*}(s)\right) \bar{D}_{2} \subset \Omega_{2}^{k_{m}} & \text { for all } m \geq s, s \in \mathbb{Z}_{+} .
\end{aligned}
$$


Step 1. Analyzing the family $\left\{\left(\phi_{k_{m}}^{2}\right)^{-1} \circ F \circ \phi_{k_{m}}^{1}\right\}_{m \in \mathbb{Z}_{+}}$.

Consider the maps $G_{m}: D_{1} \rightarrow D_{2}$ defined by

$$
G_{m}:=\left(\phi_{k_{m}}^{2}\right)^{-1} \circ F \circ \phi_{k_{m}}^{1} .
$$

By Montel's theorem, and passing to a subsequence and relabeling if necessary, we get a map $G \in \mathbb{O}\left(D_{1} ; \mathbb{C}^{n}\right)$ such that $G_{m} \rightarrow G$ uniformly on compact subsets. Let us fix an $s \in \mathbb{Z}_{+}$. By (*), we infer that there exists $M_{s} \in \mathbb{Z}_{+}$such that $(1-1 / s) \bar{D}_{j} \subset \Omega_{j}^{k_{m}}$ for all $m \geq M_{s}, j=1,2$. Note that $\left.G_{m}\right|_{\Omega_{1}^{k_{m}}}$ is a biholomorphism, whence $G_{m}^{\prime}(0)$ is invertible for each $m$. Hence, by the Schwarz lemma for convex balanced domains (i.e., Result 4.5 above) $G_{m}^{\prime}(0)$ maps $(1-1 / s) D_{1}$ into $D_{2}$ and $G_{m}^{\prime}(0)^{-1}$ maps $(1-1 / s) D_{2}$ into $D_{1}$ for all $m \geq M_{s}$. We claim that this implies that $G^{\prime}(0)$ is invertible. Suppose not. Then we would find a $z_{0}$ with $\left\|z_{0}\right\|_{1}=(1-2 / s)$ such that $G^{\prime}(0) z_{0}=0$. Note that $G_{m}^{\prime}(0) \rightarrow G^{\prime}(0)$ in norm, whence, given any $\varepsilon>0,\left\|G_{m}^{\prime}(0) z_{0}\right\|_{2}<\varepsilon$ for every sufficiently large $m$. If we now choose $\varepsilon \leq(1-2 / s)^{2}$, we see that

$$
G_{m}^{\prime}(0)^{-1}\left(\left\{\|w\|_{2}=(1-2 / s)\right\}\right) \not \subset D_{1}
$$

for all sufficiently large $m$. This is a contradiction. Hence the claim.

Now that it is established that $G^{\prime}(0)$ is invertible, it follows that $G_{m}^{\prime}(0)^{-1} \rightarrow$ $G^{\prime}(0)^{-1}$ in norm. Hence, $G^{\prime}(0)^{-1}$ maps $(1-1 / s) D_{2}$ into $D_{1}$. Recall that $s \in \mathbb{Z}_{+}$ was arbitrarily chosen and that the function $v^{*}$ in $(*)$ is strictly increasing. Thus, $G^{\prime}(0)^{-1}$ maps $D_{2}$ into $D_{1}$. By construction, $G\left(D_{1}\right) \subset \bar{D}_{2}$. Now, $D_{2}$ is complete (Kobayashi) hyperbolic. Hence $D_{2}$ is taut; see [Kiernan 1970]. As $G(0)=0 \in D_{2}$, $G$ maps $D_{1}$ to $D_{2}$. So, the holomorphic map $G^{\prime}(0)^{-1} \circ G: D_{1} \rightarrow D_{1}$ satisfies all the conditions of Cartan's uniqueness theorem. Thus,

$$
G^{\prime}(0)^{-1} \circ G=\mathrm{id}_{D_{1}},
$$

which means that $G=\left.G^{\prime}(0)\right|_{D_{1}}$.

Step 2. Showing that $D_{1}$ and $D_{2}$ are biholomorphically equivalent.

We have shown in Step 1 that $G^{\prime}(0)^{-1}$ maps $(1-1 / s) D_{2}$ into $D_{1}$. As $G^{\prime}(0)$ is injective, this means that $G^{\prime}(0)\left(D_{1}\right)$ contains $(1-1 / s) D_{2}$ for arbitrarily large $s \in \mathbb{Z}_{+}$. Thus $G$ maps $D_{1}$ onto $D_{2}$. It follows that $D_{1}$ is biholomorphic to $D_{2}$.

It would help to simplify our notation somewhat. By the nature of the argument in Step 1, it is clear that we can assume that the sequences $\left\{a_{k}\right\} \subset \Omega_{1}$ and $\left\{b_{k}\right\} \subset \Omega_{2}$ are so selected that $(*)$ is true with $\left\{k_{m}\right\}_{m \in \mathbb{Z}_{+}}=\{1,2,3, \ldots\}$. Owing to Step 2, we may now assume $D_{1}=D_{2}=: D$. The argument we will make in Step 3 below is valid regardless of the specific sequence $\left\{a_{k}\right\}$ or $\left\{b_{k}\right\}$. Hence, in the next three paragraphs following this, the sequence $\left\{A_{k}\right\}$ will stand for either $\left\{a_{k}\right\}$ or $\left\{b_{k}\right\}$, and the point $q$ will stand for either $p$ or $F(p)$. Also, we will abbreviate $\phi_{A_{k}}^{j}$ to $\phi_{k}$. 
Step 3. Producing subsequences of $\left\{\phi_{k}\right\}$ that converge on "large" subsets of $\partial D$. By Result 4.3 we may take $\phi_{k}=g_{A_{k}}$, whence

$$
\phi_{k}^{\prime}(z)=\mathrm{B}_{D}\left(A_{k}, A_{k}\right)^{1 / 2} \circ \mathrm{B}_{D}\left(-z, A_{k}\right)^{-1} .
$$

In the argument that follows, it is implicit that each $\phi_{k}$ is defined as a holomorphic map on some neighborhood (which depends on $\phi_{k}$ ) of $\bar{D}$; see Lemma 4.2. By Proposition 4.7 we can find a point $\xi_{0} \in M_{D, 1}$ such that

$$
\operatorname{det} \mathrm{B}_{D}\left(e^{i \theta} \xi_{0}, q\right) \neq 0 \quad \text { for all } \theta \in \mathbb{R} .
$$

By continuity, there exist a $\bar{D}$-open neighborhood $\Gamma$ of $q$, an $\mu_{D, 1}$-open neighborhood $W$ of $\xi_{0}$, and a $\bar{D}$-open set $V$ with the following properties:

(a) $z \in V \Longrightarrow e^{i \theta} z \in V$ for all $\theta \in \mathbb{R}$,

(b) $V \cap \partial D=S^{1} \cdot W$,

(c) $z \in V \Longrightarrow t z \in V$ for all $t \in[1,1 /\|z\|]$

(now $\|\cdot\|$ is the spectral norm associated to $D$ ), such that

$$
\operatorname{det} \mathrm{B}_{D}(z, w) \neq 0 \text { for all }(z, w) \in \bar{V} \times \Gamma \text {. }
$$

Here, given a set $X \subset \mathbb{C}^{n}, S^{1} \cdot X$ stands for the set $\left\{e^{i \theta} x: x \in X, \theta \in \mathbb{R}\right\}$. Let us call any pair $(V, W)$, where $V$ is a $\bar{D}$-open set and $W$ is an $\mu_{D, 1}$-open set, a truncated prism with base $S^{1} \cdot W$ if ( $\left.V, W\right)$ satisfies properties (a)-(c) above.

We can find $V^{\prime}$ and $W^{\prime}$, with $\bar{W}^{\prime} \subset W$, such that $\left(V^{\prime}, W^{\prime}\right)$ is a truncated prism with base $S^{1} \cdot W^{\prime}$ with the properties

- $\bar{V}^{\prime} \subset V$;

- there exists a $\delta_{0} \ll 1$ such that for $z_{1}, z_{2} \in \bar{V}^{\prime}$, the segment $\left[z_{1}, z_{2}\right] \subset V$ whenever $\left|z_{1}-z_{2}\right|<\delta_{0}$.

Owing to holomorphicity and convexity,

$$
\phi_{k}\left(z_{1}\right)-\phi_{k}\left(z_{2}\right)=\int_{0}^{1} \phi_{k}^{\prime}\left(z_{1}+t\left(z_{2}-z_{1}\right)\right)\left(z_{2}-z_{1}\right) d t, \quad z_{1}, z_{2} \in \bar{D} .
$$

We can find a $K \equiv K(W)$ such that, in view of (5-3), $\left\{\mathrm{B}_{D}\left(z, A_{k}\right): k \geq K(W), z \in \bar{V}\right\}$ is a compact family in $\operatorname{GL}(n, \mathbb{C})$. Hence, in view of (5-2) (and since $\left\{\mathrm{B}_{D}\left(A_{k}, A_{k}\right)\right.$ : $\left.k \in \mathbb{Z}_{+}\right\}$is a relatively compact family in $\left.\mathbb{C}^{n \times n}\right)$, there exists a constant $C>0$ such that

$$
\left\|\phi_{k}^{\prime}(z)\right\|_{\text {op }} \leq C \quad \text { for all } z \in \bar{V} \text { and } k \geq K .
$$

By our construction of $V^{\prime}$ and from (5-4), we conclude that

$$
\left|\phi_{k}\left(z_{1}\right)-\phi_{k}\left(z_{2}\right)\right| \leq C\left|z_{1}-z_{2}\right| \text { for all } z_{1}, z_{2} \in \bar{V}^{\prime},\left|z_{1}-z_{2}\right|<\delta_{0} \text {, and } k \geq K \text {. }
$$

In short, $\left\{\left.\phi_{k}\right|_{\bar{V}^{\prime}}\right\} \subset \mathscr{C}\left(\bar{V}^{\prime} ; \mathbb{C}^{n}\right)$ is an equicontinuous family. 
By the Arzelà-Ascoli theorem, we can find a subsequence of $\left\{\phi_{k}\right\}$ that converges uniformly to $q$ on $\bar{V}^{\prime}$. For simplicity of notation, let us continue to denote this subsequence by $\left\{\phi_{k}\right\}$. Then there exists a $K_{1} \in \mathbb{Z}_{+}$such that $\phi_{k}\left(\bar{V}^{\prime}\right) \subset \Omega$ (which denotes either $\Omega_{1}$ or $\Omega_{2}$ ) for all $k \geq K_{1}$. Furthermore, we may assume that $K_{1}$ is so large that, thanks to $(*)$,

$$
(1-1 / s) \bar{D} \subset \phi_{k}^{-1}(\Omega) \text { for all } k \geq K_{1},
$$

where $s$ is so large that $(1-1 / s) \bar{D} \cap V^{\prime}$ is a nonempty open set. By construction,

$$
z \in V^{\prime} \cap D \Longrightarrow \Delta_{z} \subset(1-1 / s) \bar{D} \cup V^{\prime} .
$$

Hence $\Delta_{z} \subset \phi_{k}^{-1}(\Omega)$ for all $k \geq K_{1}$. We summarize this paragraph as follows:

(**) Given any truncated prism $(V, W)$ with base $S^{1} \cdot W$ such that $\mathrm{B}_{D}\left(z, A_{k}\right) \neq 0$ on $\bar{V}$ for all $k$ sufficiently large, we can find a $K_{1} \in \mathbb{Z}_{+}$and a truncated prism $\left(V^{\prime}, W^{\prime}\right)$ with $\bar{V}^{\prime} \subset V$ such that $\Delta_{z} \subset \phi_{k}^{-1}(\Omega)$ for each $z \in V^{\prime} \cap D$ and each $k \geq K_{1}$.

\section{Step 4. Completing the proof.}

By Proposition 4.7 and $(* *)$, we can find a truncated prism $\left(V^{\prime}, W^{\prime}\right)$ with base $S^{1} \cdot W^{\prime}$ which has all the properties stated in $(* *)$. Let $s \in \mathbb{Z}_{+}$be so large that $(1-1 / s) D \cap V^{\prime}:=U^{\prime}$ is a nonempty open set. As $G_{k} \rightarrow G$ uniformly on $U^{\prime}$ (by Step 1), there exist a point $w_{0} \in G\left(U^{\prime}\right), K_{2} \in \mathbb{Z}_{+}$and a $c>0$ such that

$$
B\left(w_{0}, c\right) \subset G\left(U^{\prime}\right) \cap G_{k}\left(U^{\prime}\right) \quad \text { and } \quad B\left(w_{0}, c\right) \subset \Omega_{2}^{k} \quad \text { for all } k \geq K_{2} .
$$

Write $\|\cdot\|$ for the spectral norm associated to $D$. Let $R: \mathbb{C}^{n} \backslash 0 \rightarrow \partial D$ be given by $R(w):=w /\|w\|$. By Proposition 4.7 and (**), we can find an $M_{D, 1}$-open subset $\omega_{2}$ such that

$$
\omega_{2} \subset R\left(B\left(w_{0}, c\right)\right),
$$

a truncated prism $\left(V_{2}, \omega_{2}\right)$ with base $S^{1} \cdot \omega_{2}$, and a $K_{3} \in \mathbb{Z}_{+}$such that $\Delta_{w} \subset \Omega_{2}^{k}$ for each $w \in V_{2} \cap D$ and each $k \geq K_{3}$. Let us now set $U:=G^{-1}\left(R^{-1}\left(\omega_{2}\right) \cap B\left(w_{0}, c\right)\right)$, and $K^{*}:=\max \left(K_{1}, K_{2}, K_{3}\right)$. Finally, we set

$$
W_{j}:=\left(\phi_{K^{*}}^{j}\right)^{-1}\left(\Omega_{j}\right), \quad j=1,2,
$$

with the understanding that $\phi_{k}^{1}=g_{a_{k}}$ and $\phi_{k}^{2}=g_{b_{k}}$.

As $U \subset V^{\prime}$, we have $\Delta_{z} \subset W_{1}$ for each $z \in U$. By construction,

$$
G_{K^{*}}(z) \in B\left(w_{0}, c\right) \subset W_{2} \text { for all } z \in U .
$$

Finally, by construction, for each $z \in U$, there exists a point $w_{z} \in \Delta_{G_{K^{*}}(z)}$ that belongs to $V_{2} \cap D$. Thus, $\Delta_{G_{K^{*}}(z)} \subset W_{2}$. Recall that $\left.G_{K^{*}}\right|_{W_{1}}: W_{1} \rightarrow W_{2}$ is a biholomorphism and $G_{K^{*}}(0)=0$. By our Key Lemma $1.2, G_{K^{*}}$, and consequently $F$, must be a biholomorphism. 


\section{The proof of Theorem 1.5}

As $p$ is an orbit accumulation point, there is a point $a_{0} \in D_{1}$ and a sequence $\left\{\phi_{k}\right\} \subset \operatorname{Aut}\left(D_{1}\right)$ such that $\phi_{k}\left(a_{0}\right) \rightarrow p$. Regardless of whether $p$ is a peak point or $F(p)$ is a peak point, let us denote the relevant peak function as $H$. Let $B$ denote a small ball centered at $p$, with $B \Subset U$, if $p$ is a peak point, and centered at $F(p)$, with $B \Subset F(U)$, if $F(p)$ is a peak point. Depending on whether $p$ or $F(p)$ is a peak point, set $G:=F^{-1}$ or $G:=F$, respectively. Finally, set

$$
h:= \begin{cases}\left.H \circ G\right|_{\overline{B \cap D_{2}}} & \text { if } p \text { is a peak point, } \\ \left.H \circ G\right|_{\overline{B \cap D_{1}}} & \text { if } F(p) \text { is a peak point. }\end{cases}
$$

By our hypothesis on $F$, it follows that $h$ satisfies all the conditions required of the function $h$ in Lemma 4.4 for the appropriate choice of $(D, p)$ depending on whether $p$ or $F(p)$ is a peak point.

Let us now denote the automorphisms discussed above by $\phi_{k}^{1}, k=1,2,3, \ldots$. Then, using $H$ or the function $h$ constructed above, depending on whether $p$ or $F(p)$ is a peak point, we deduce by Lemma 4.4 that $\phi_{k}^{1} \rightarrow$ const $_{p}$ uniformly on compact subsets of $D_{1}$. Set $a_{k}:=\phi_{k}^{1}(0)$. As $a_{k} \rightarrow p$, we may assume without loss of generality that $a_{k} \in U$. Let $b_{k}:=F\left(a_{k}\right)$, and let $\phi_{k}^{2} \in \operatorname{Aut}\left(D_{2}\right)$ be an automorphism that maps 0 to $b_{k}$ (which is possible as $\operatorname{Aut}\left(D_{2}\right)$ acts transitively on $D_{2}$ ). Repeating the above argument, $\phi_{k}^{2} \rightarrow$ const $_{F(p)}$ uniformly on compact subsets of $D_{2}$. We have arrived at the same result as in (5-1). Thereafter, if we define

$$
\Omega_{j}^{k}:=\left(\phi_{k}^{j}\right)^{-1}\left(\Omega_{j}\right), \quad j=1,2, k \in \mathbb{Z}_{+},
$$

where $\Omega_{1}:=U$ and $\Omega_{2}:=F(U)$, then, reasoning exactly as in the passage following (5-1), we deduce that $(*)$ from Section 5 holds true for our present setup.

With $\left\{k_{m}\right\}_{m \in \mathbb{Z}_{+}}$as given by $(*)$, let us define the maps $G_{m}: \Omega_{1}^{k_{m}} \rightarrow \Omega_{2}^{k_{m}}$ by

$$
G_{m}:=\left(\phi_{k_{m}}^{2}\right)^{-1} \circ F \circ \phi_{k_{m}}^{1} .
$$

By construction, each $G_{m}$ is a biholomorphic map. In particular,

$$
G_{m}(0)=0 \quad \text { and } \quad G_{m}^{\prime}(0) \in \operatorname{GL}(n, \mathbb{C}) .
$$

We may assume, owing to (5-1), that the sequences $\left\{\Omega_{j}^{k_{m}}\right\}_{m \in \mathbb{Z}_{+}}$are increasing sequences. By Montel's theorem, and arguing by induction, we can find sequences $\left\{G_{l, m}\right\}$ and holomorphic maps $\Gamma_{l}: \Omega_{1}^{k_{l}} \rightarrow \bar{D}_{2}$ such that for $l=1,2,3, \ldots$,

- $\left\{G_{1, m}\right\}_{m \in \mathbb{Z}_{+}}$is a subsequence of $\left\{G_{\nu}\right\}_{v \in \mathbb{Z}_{+}}$and $\left\{G_{l+1, m}\right\}_{m \in \mathbb{Z}_{+}}$is a subsequence of $\left\{G_{l, v}\right\}_{v \in \mathbb{Z}_{+}}$;

- $\left.G_{l, m}\right|_{\Omega_{1}^{k_{l}}} \rightarrow \Gamma_{l}$ as $m \rightarrow \infty$, uniformly on compact subsets of $\Omega_{1}^{k_{l}}$. 
Owing to this construction, the rule

$$
\Gamma(z):=\Gamma_{l}(z) \quad \text { if } z \in \Omega_{1}^{k_{l}}
$$

gives a well-defined holomorphic map $\Gamma: D_{1} \rightarrow \bar{D}_{2}$.

Let us define $H_{l}:=G_{l, l}$. Now suppose $\Gamma\left(D_{1}\right) \cap \partial D_{2} \neq \varnothing$. Then, there exists $\xi \in D_{1}$ such that $\Gamma(\xi) \in \partial D_{2}$. Let $M \in \mathbb{Z}_{+}$be so large that $\Omega_{1}^{k_{M}} \ni \xi$. As $D_{2}$ is a bounded symmetric domain, it is taut. Thus, by focusing attention on the sequence

$$
\left\{\left.H_{l}\right|_{\Omega_{1}^{k_{M}}}: l=M, M+1, M+2, \ldots\right\} \subset \mathcal{O}\left(\Omega_{1}^{k_{M}} ; D_{2}\right),
$$

we conclude, by assumption, that $\Gamma\left(\Omega_{1}^{k_{M}}\right) \subset \partial D_{2}$. But, by (6-1), $\Gamma(0)=0 \notin \partial D_{2}$. This is a contradiction, from which we infer:

(a) The range of $\Gamma$ is a subset of $D_{2}$.

Now observe that, by $(*)$, we have:

(b) The sequence $\left\{H_{l}: l=s, s+1, s+2, \ldots\right\}$ converges uniformly to $\Gamma$ on $(1-1 / s) \bar{D}_{1}, s \in \mathbb{Z}_{+}$;

(c) $H_{l}^{-1}$ maps 0 to 0 and $\left(1-1 / \nu^{*}(l)\right) D_{2}$ into $D_{1}$ (since $\operatorname{dom}\left(H_{l}^{-1}\right)=\operatorname{range}\left(H_{l}\right) \supseteq$ $\left.\Omega_{2}^{k_{l}}\right)$.

In view of (6-1) and the fact that $D_{1}$ and $D_{2}$ are balanced, (a)-(c) are precisely the ingredients required to repeat the argument in Step 1 of the proof of Theorem 1.1 to infer that $\Gamma^{\prime}(0)$ is invertible, $\Gamma^{\prime}(0)^{-1}: D_{2} \rightarrow D_{1}$ and

$$
\Gamma^{\prime}(0)^{-1} \circ \Gamma=\mathrm{id}_{D_{1}} \text {. }
$$

Thus, by (a), $\Gamma^{\prime}(0)\left(D_{1}\right) \subset D_{2}$. One of the consequences of repeating the argument contained in Step 1 in Theorem 1.1 is, in view of (c), that $\Gamma^{\prime}(0)^{-1}$ maps $\left(1-1 / v^{*}(l)\right) D_{2}$ into $D_{2}$ for every $l \in \mathbb{Z}_{+}$. As $v^{*}$ is strictly increasing and $\mathbb{Z}_{+}$-valued, and as $\Gamma^{\prime}(0)$ is injective, this means that $\Gamma^{\prime}(0)\left(D_{1}\right)$ contains $(1-1 / s) D_{2}$ for arbitrarily large $s \in \mathbb{Z}_{+}$, whence $\Gamma^{\prime}(0)$ maps $D_{1}$ onto $D_{2}$. Hence, $\left.\Gamma^{\prime}(0)\right|_{D_{1}}$ is a biholomorphism of $D_{1}$ onto $D_{2}$.

\section{Acknowledgements}

Jaikrishnan Janardhanan would like to thank his colleagues and friends G.P. Balakumar, Dheeraj Kulkarni, Divakaran Divakaran and Pranav Haridas for many interesting discussions. Gautam Bharali contributed to this work while on sabbatical at the Norwegian University of Science and Technology (NTNU) in Trondheim. He would like to acknowledge the support and the hospitality of the Department of Mathematical Sciences at NTNU. He also thanks John Erik Fornæss for his helpful comments on this work. 


\section{References}

[Alexander 1977] H. Alexander, "Proper holomorphic mappings in $C^{n}$ ", Indiana Univ. Math. J. 26:1 (1977), 137-146. MR 54 \#10685 Zbl 0391.32015

[Bell 1982] S. R. Bell, "Proper holomorphic mappings between circular domains", Comment. Math. Helv. 57:4 (1982), 532-538. MR 84m:32032 Zbl 0511.32013

[Bell 1993] S. Bell, "Algebraic mappings of circular domains in $\mathbb{C}^{n}$ ", pp. 126-135 in Several complex variables (Stockholm, 1987/1988), edited by J. E. Fornæss, Math. Notes 38, Princeton Univ. Press, 1993. MR 94a:32040 Zbl 0774.32001

[Chern and Moser 1974] S. S. Chern and J. K. Moser, "Real hypersurfaces in complex manifolds", Acta Math. 133 (1974), 219-271. MR 54 \#13112 Zbl 0302.32015

[Harish-Chandra 1956] Harish-Chandra, "Representations of semisimple Lie groups, VI: Integrable and square-integrable representations", Amer. J. Math. 78 (1956), 564-628. MR 18,490d Zbl 0072.01702

[Henkin and Novikov 1984] G. M. Henkin and R. G. Novikov, "Proper mappings of classical domain", pp. 625-627 in Linear and complex analysis problem book: 199 research problems, edited by V. P. Havin et al., Lecture Notes in Mathematics 1043, Springer, Berlin, 1984. MR 85k:46001 Zbl 0545.30038

[Kerzman and Rosay 1981] N. Kerzman and J.-P. Rosay, "Fonctions plurisousharmoniques d'exhaustion bornées et domaines taut”, Math. Ann. 257:2 (1981), 171-184. MR 83g:32019 Zbl 0451.32012

[Kiernan 1970] P. Kiernan, "On the relations between taut, tight and hyperbolic manifolds", Bull. Amer. Math. Soc. 76 (1970), 49-51. MR 40 \#5896 Zbl 0192.44103

[Koecher 1999] M. Koecher, The Minnesota notes on Jordan algebras and their applications, Lecture Notes in Mathematics 1710, Springer, Berlin, 1999. MR 2001e:17040 Zbl 1072.17513

[Korányi 1969] A. Korányi, "Harmonic functions on Hermitian hyperbolic space", Trans. Amer. Math. Soc. 135 (1969), 507-516. MR 43 \#3480 Zbl 0174.38801

[Łojasiewicz 1959] S. Łojasiewicz, "Sur le problème de la division”, Studia Math. 18 (1959), 87-136. MR 21 \#5893 Zbl 0115.10203

[Loos 1977] O. Loos, "Bounded symmetric domains and Jordan pairs", Mathematical Lectures, University of California, Irvine, 1977.

[Mok and Tsai 1992] N. Mok and I. H. Tsai, "Rigidity of convex realizations of irreducible bounded symmetric domains of rank $\geq 2$ ", J. Reine Angew. Math. 431 (1992), 91-122. MR 93h:32046 Zbl 0765.32017

[Narasimhan 1971] R. Narasimhan, Several complex variables, The University of Chicago Press, Chicago, IL, 1971. MR 49 \#7470 Zbl 0223.32001

[Roos 2000] G. Roos, "Jordan triple systems", pp. 425-536 in Analysis and geometry on complex homogeneous domains, Progress in Mathematics 185, Birkhäuser, Boston, MA, 2000. MR 2001f:32036 Zbl 1043.17017

[Rudin 1980] W. Rudin, Function theory in the unit ball of $\mathbf{C}^{n}$, Grundlehren der Mathematischen Wissenschaften 241, Springer, New York, 1980. MR 82i:32002 Zbl 0495.32001

[Tsai 1993] I. H. Tsai, "Rigidity of proper holomorphic maps between symmetric domains", $J$. Differential Geom. 37:1 (1993), 123-160. MR 93m:32038 Zbl 0799.32027

[Tu 2002] Z.-H. Tu, "Rigidity of proper holomorphic mappings between equidimensional bounded symmetric domains", Proc. Amer. Math. Soc. 130:4 (2002), 1035-1042. MR 2003a:32027 Zbl 0999.32007 
[Tumanov and Khenkin 1982] A. E. Tumanov and G. M. Khenkin, "Local characterization of analytic automorphisms of classical domains”, Dokl. Akad. Nauk SSSR 267:4 (1982), 796-799. In Russian; translated in Sov. Math. Dokl. 26 (1982), 702-705. MR 85b:32048 Zbl 0529.32014

[Vigué 1991] J.-P. Vigué, "Un lemme de Schwarz pour les domaines bornés symétriques irréductibles et certains domaines bornés strictement convexes”, Indiana Univ. Math. J. 40:1 (1991), 293-304. MR 92c:32026 Zbl 0733.32025

[Wolf 1972] J. A. Wolf, "Fine structure of Hermitian symmetric spaces", pp. 271-357 in Symmetric spaces: Short courses (St. Louis, MO, 1969-1970), edited by W. M. Boothby and G. L. Weiss, Pure and App. Math. 8, Dekker, New York, 1972. MR 53 \#8516 Zbl 0257.32014

[Wolf and Korányi 1965] J. A. Wolf and A. Korányi, "Generalized Cayley transformations of bounded symmetric domains”, Amer. J. Math. 87 (1965), 899-939. MR 33 \#229 Zbl 0137.27403

Received June 10, 2013.

\section{GAUTAM BHARALI}

DEPARTMENT OF MATHEMATICS

INDIAN INSTITUTE OF SCIENCE

BANGALORE 560012

INDIA

bharali@math.iisc.ernet.in

JAIKRISHNAN JANARDHANAN

DEPARTMENT OF MATHEMATICS

INDIAN INSTITUTE OF SCIENCE

BANGALORE 560012

INDIA

jaikrishnan@math.iisc.ernet.in 


\title{
PACIFIC JOURNAL OF MATHEMATICS
}

\author{
msp.org/pjm
}

Founded in 1951 by E. F. Beckenbach (1906-1982) and F. Wolf (1904-1989)

\section{EDITORS}

Don Blasius (Managing Editor)

Department of Mathematics

University of California

Los Angeles, CA 90095-1555

blasius@math.ucla.edu

\author{
Paul Balmer \\ Department of Mathematics \\ University of California \\ Los Angeles, CA 90095-1555 \\ balmer@math.ucla.edu \\ Robert Finn \\ Department of Mathematics \\ Stanford University \\ Stanford, CA 94305-2125 \\ finn@math.stanford.edu \\ Sorin Popa \\ Department of Mathematics \\ University of California \\ Los Angeles, CA 90095-1555 \\ popa@math.ucla.edu
}

\author{
Vyjayanthi Chari \\ Department of Mathematics \\ University of California \\ Riverside, CA 92521-0135 \\ chari@math.ucr.edu \\ Kefeng Liu \\ Department of Mathematics \\ University of California \\ Los Angeles, CA 90095-1555 \\ liu@math.ucla.edu \\ Jie Qing \\ Department of Mathematics \\ University of California \\ Santa Cruz, CA 95064 \\ qing@ cats.ucsc.edu
}

\section{PRODUCTION}

Silvio Levy, Scientific Editor, production@msp.org

\section{SUPPORTING INSTITUTIONS}

ACADEMIA SINICA, TAIPEI

CALIFORNIA INST. OF TECHNOLOGY

INST. DE MATEMÁTICA PURA E APLICADA

KEIO UNIVERSITY

MATH. SCIENCES RESEARCH INSTITUTE

NEW MEXICO STATE UNIV.

OREGON STATE UNIV.

\author{
STANFORD UNIVERSITY \\ UNIV. OF BRITISH COLUMBIA \\ UNIV. OF CALIFORNIA, BERKELEY \\ UNIV. OF CALIFORNIA, DAVIS \\ UNIV. OF CALIFORNIA, LOS ANGELES \\ UNIV. OF CALIFORNIA, RIVERSIDE \\ UNIV. OF CALIFORNIA, SAN DIEGO \\ UNIV. OF CALIF., SANTA BARBARA
}

\author{
Daryl Cooper \\ Department of Mathematics \\ University of California \\ Santa Barbara, CA 93106-3080 \\ cooper@math.ucsb.edu \\ Jiang-Hua Lu \\ Department of Mathematics \\ The University of Hong Kong \\ Pokfulam Rd., Hong Kong \\ jhlu@maths.hku.hk \\ Paul Yang \\ Department of Mathematics \\ Princeton University \\ Princeton NJ 08544-1000 \\ yang@math.princeton.edu
}

These supporting institutions contribute to the cost of publication of this Journal, but they are not owners or publishers and have no responsibility for its contents or policies.

See inside back cover or msp.org/pjm for submission instructions.

The subscription price for 2014 is US $\$ 410 /$ year for the electronic version, and \$535/year for print and electronic.

Subscriptions, requests for back issues and changes of subscribers address should be sent to Pacific Journal of Mathematics, P.O. Box 4163, Berkeley, CA 94704-0163, U.S.A. The Pacific Journal of Mathematics is indexed by Mathematical Reviews, Zentralblatt MATH, PASCAL CNRS Index, Referativnyi Zhurnal, Current Mathematical Publications and Web of Knowledge (Science Citation Index).

The Pacific Journal of Mathematics (ISSN 0030-8730) at the University of California, c/o Department of Mathematics, 798 Evans Hall \#3840, Berkeley, CA 94720-3840, is published twelve times a year. Periodical rate postage paid at Berkeley, CA 94704, and additional mailing offices. POSTMASTER: send address changes to Pacific Journal of Mathematics, P.O. Box 4163, Berkeley, CA 94704-0163.

PJM peer review and production are managed by EditFLOW ${ }^{\circledR}$ from Mathematical Sciences Publishers.

\section{PUBLISHED BY}

\section{mathematical sciences publishers \\ nonprofit scientific publishing}

http://msp.org/

(C) 2014 Mathematical Sciences Publishers 


\section{PACIFIC JOURNAL OF MATHEMATICS}

Volume $271 \quad$ No. $1 \quad$ September 2014

Proper holomorphic maps between bounded symmetric domains revisited 1

GAUTAM BHARALI and JAIKRISHNAN JANARDHANAN

An explicit Majorana representation of the group $3^{2}: 2$ of $3 C$-pure type 25

HSIAN-YANG CHEN and CHING HUNG LAM

Sofic groups: graph products and graphs of groups

LAUra Ciobanu, DereK F. Holt and SARAh ReES

Perturbations of a critical fractional equation

Eduardo Colorado, Arturo de Pablo and Urko SÁnchez

A density theorem in parametrized differential Galois theory

THOMAS DREYFUS

On the classification of complete area-stationary and stable surfaces in $\quad 143$ the subriemannian Sol manifold

MATteo Galli

Periodic orbits of Hamiltonian systems linear and hyperbolic at infinity

BAŞAK Z. GÜREL

Nonsplittability of the rational homology cobordism group of

3-manifolds

SE-Goo KIM and CHARLES LIVINGSTON

Biharmonic surfaces of constant mean curvature

ERIC LOUBEAU and CEZAR ONICIUC

Foliations of a smooth metric measure space by hypersurfaces with constant $f$-mean curvature

JUNCHEOL PYO

On the existence of large degree Galois representations for fields of small 243 discriminant

JEREMY ROUSE and FRANK THORNE 